\title{
Behandlung der respiratorischen Papillomatose - Kasuistik zur systemischen Therapie mit Bevacizumab ${ }^{1}$
}

\author{
Treatment of Respiratory Papillomatosis - A Case Report on Systemic \\ Treatment with Bevacizumab
}

Autoren

Institut

\author{
S. Nagel, C. Busch, T. Blankenburg, W. Schütte
}

Krankenhaus Martha-Maria Halle-Dölau gGmbH, Klinik für Innere Medizin II (CA PD Dr. med. W. Schütte), Halle eingereicht 18.12.2008 akzeptiert nach Revision 14. 4.2009

\section{Bibliografie}

DOI $10.1055 / \mathrm{s}-0029-1214714$

Pneumologie 2009; 63:

387-389 @ Georg Thieme Verlag KG Stuttgart · New York ISSN 0934-8387

\section{Korrespondenzadresse}

Dr. med. Sylke Nagel

Krankenhaus Martha-Maria Halle-Dölau gGmbH

Klinik für Innere Medizin II

Röntgenstr. 1

06120 Halle

S.Nagel@MarthaMaria-Halle.de

\section{Zusammenfassung \\ $\nabla$}

Hintergrund: Die rezidivierende respiratorische Papillomatose (RRP) ist ein seltenes Krankeitsbild, das durch humane Papillomaviren hervorgerufen wird und charakterisiert ist durch multiple exophytische Läsionen und deren unkontrolliertes Wachstum innerhalb des Respirationstraktes. Häufigste Komplikation der RRP ist die Stenosierung der Trachea. Medikamentöse Therapieoptionen sind bislang wenig effektiv. Die Inhibition des vaskulären endothelialen Wachstumsfaktors (VEGF) durch Bevacizumab könnte eine erfolgversprechende Therapieoption bei RRP sein.

Kasustik: Es wird über einen 32-jährigen männlichen Patienten mit RRP berichtet, der seit 1996 vierteljährlich wegen symptomatischer Trachealstenose behandelt wird. Die einzig wirksame Behandlung war die Laserkoagulation. Andere Therapien zeigten keinen Effekt. Im Mai 2006 wurden intrapulmonale Läsionen der RRP diagnostiziert, ohne Nachweis von Malignität. Von Dezember 2007 bis Juni 2008 wurde der Patient mit Bevacizumab behandelt. Darunter war eine deutliche Remission der RRP sowie der Symptomatik zu beobachten. Unter dieser Behandlung war keine zusätzliche Laserabtragung notwendig.

Schlussfolgerung: Die VEGF-Hemmung durch Bevacizumab scheint eine neue effektive Option im Krankheitsmanagement der RRP zu bieten.

\section{Abstract \\ $\nabla$}

Background: Recurrent respiratory papillomatosis (RRP) is a known but rare disease, caused by human papilloma virus and characterised by multiple exophytic lesions and uncontrolled growth of papilloma in the respiratory tract. The most common complication of RRP is stenosis of the trachea. Medical therapeutic options have so far been less effective. However, inhibition of vascular endothelial growth factor (VEGF) by bevacizumab does appear to be an effective treatment option for RRP.

Case Report: The case of a 32-year-old male patient with RRP who has been treated for his symptomatic tracheal stenosis four times a year since 1996 is described. Only treatment by laser ablation showed any efficacy. Alternative treatment options did not show any effect. In May 2006 intrapulmonary lesions of RRP were also diagnosed but without any malignancy. From December 2007 to June 2008 the patient has been treated with bevacizumab. A visible regression of RRP and markedly less symptoms were observed. During this treatment no further laser ablation was necessary.

Conclusion: Inhibition of VEGF by bevacizumab seems to offer a new and effective option in the medical management of RRP.

\section{Einleitung}

$\nabla$

Die rezidivierende respiratorische Papillomatose (RRP) ist ein seltenes Krankheitsbild, welches bei 2 von 100000 Erwachsenen und bei 4 von 100000 Kindern auftritt [1]. Man unterscheidet

\footnotetext{
${ }^{1}$ Posterpräsentation, 50. Tagung der Deutschen Gesellschaft für Pneumologie und Beatmungsmedizin, 18. - 21 März 2009.
}

eine juvenile und eine adulte Form. Die juvenile Form tritt in den ersten Lebensjahren oder im Teenager-Alter auf. Die Erwachsenenform manifestiert sich in der 3. oder 4. Lebensdekade. Humane Papillomaviren wie HPV 6 und 11 sind ursächlich für das Entstehen der RRP anzusehen [2]. Obwohl die RRP eine benigne Erkrankung ist, so hat sie doch einen signifikanten Krankheitswert und führt in einzelnen Fällen durch rezidivierendes Auftreten zum Tode. 
Je disseminierter und ausgebreiteter die Papillomatose im Respirationstrakt auftritt, um so schlechter ist die Prognose. Der Verlauf der Erkrankung ist variabel, d. h. die Papillome können regredient sein, persistieren oder in wenigen Beispielen auch einen Progress zeigen mit Transformation zu einem Karzinom [3].

Je eher sich die RRP klinisch manifestiert, umso höher ist der Risikofaktor für eine Streuung der Papillomatose in das distale Bronchialsystem [3,4]. Die für die RRP ursächlichen humanen Papillomaviren Typ 6 und 11 sind hauptsächlich im menschlichem anorectal-genitalen Trakt zu finden. Im genitalen Bereich verursachen die Viren genitale Infektionen bzw. Warzen, deren Inzidenz jährlich ansteigt [5].

Dies führt zu der Vermutung, dass ein erhöhtes Risiko besteht, wenn eine genitale HPV-Infektion von der Mutter auf das Kind unter der Geburt übertragen wird. Diese Hypothese wurde von Silverberg et al. unterstützt. Sie zeigten, dass bei Schwangeren mit genitaler HPV-Infektion ein 200-fach erhöhtes Risiko bestand, dass deren Kinder an einer RRP erkranken [6]. Bei der adulten Form der RRP werden ursächlich für deren Genese ein häufiger Wechsel der Sexualpartner sowie oraler Sex vermutet [7].

Die Papillome der RRP wachsen bevorzugt an „Transformationszonen“, wo Plattenepithel in Flimmerepithel übergeht [8]. Interessanterweise wurden Papillome auch beobachtet an Stellen von Tracheotomien, an denen iatrogen eine Veränderung der Epithelzone hervorgerufen wurde [9]. Die Viren HPV 6 und 11 treten in die Wirtszelle, den basalen Keratinozyten, infolge Mikrotraumata oder Oberflächendefekte des Epithels ein. Dabei spielen vermutlich bestimmte Rezeptoren eine Rolle [10,11]. Innerhalb der infizierten Wirtszelle erfolgt die Replikation des Virus. Die infizierten Keratinozyten proliferieren und wachsen in einem veränderten Wachstumsmuster, was zur Bildung von Papillomen führt [12]. Diese Papillome wachsen in das Lumen des Tracheobronchialtraktes und engen dieses ein. Die Entfernung mittels Laserablation oder Argon-Plasma-Koagulation der Papillome ist Therapie der Wahl. Dadurch kann eine Wiedereröffnung der Luftwege hergestellt werden sowie eine Verbesserung der Symptome und der Lebensqualität erzielt werden [13].

Da das HPV in normalen Mukosazellen persistiert, muss immer wieder mit einem Rezidiv der RRP gerechnet werden [14]. Gehäufte lokale Therapie, wie Lasertherapien oder Behandlung führen jedoch zu Veränderungen der Trachea und Vernarbungen des Respirationstraktes und letztendlich zu Stenosen [14]. Andere Therapieoptionen sind die Gabe von Virostatika (Cidofovir), welches in die Papillome injiziert wird oder die Gabe von Ribavirin oder von Mumpsvakzinen oder die photodynamische Therapie [15]. Bislang konnte in kontrollierten Studien kein Beweis erbracht werden, dass eine Behandlung mit antiviralen Agenzien als adjuvante Therapie suffizient dem Management der RRP dient. Ein möglicher neuer Therapieansatz zur Behandlung der RRP ist die Inhibition des vaskulären endothelialen Wachstumsfaktors (VEGF). Bislang wird diese Behandlung bei malignen Tumoren, wie dem Lungenkarzinom oder dem Kolonkarzinom, angewandt. Die Inhibitoren (z. B. der Antikörper Bevacizumab) binden an VEGF. Dadurch wird die Neoangiogenese eingeschränkt und es kommt zur Wachstumshemmung bzw. zur Schrumpfung des Tumors [16].

In einer retrospektiven Analyse konnten Rahbar et al. bei Patienten mit RRP eine starke Expression von VEGF-A mRNA an den Epithelien der Papillome sowie von VEGFR-1 und VEGFR-2 mRNAs an den Endothelzellen der subepithelialen Blutgefäße feststellen [17]. Dies lässt vermuten, dass VEGF in der Pathogene- se der RRP eine Rolle spielt und die Anwendung von Bevacizumab als VEGF-Inhibitor ein möglicher Therapieansatz zur Rezidivhemmung der RRP darstellt.

In der vorliegenden Kasuistik wird über einen 32 Jahre alten Patienten mit einer RRP berichtet, bei dem ein Therapieversuch mit dem VEGF-Inhibitor Bevacizumab vorgenommen wurde.

\section{Kasuistik \\ $\nabla$}

\section{Anamnese und Untersuchungsbefunde}

Ein in diesem Jahr 32-jährig gewordener junger männlicher Patient erlitt im Alter von 2 Jahren eine akute respiratorische Insuffizienz aufgrund einer laryngealen Papillomatose. Es erfolgte daraufhin die Tracheotomie. Aufgrund der ständigen Rezidive wurde die Tracheostomaanlage für 10 Jahre belassen. Während dieser Zeit wurde er HNO-ärztlich behandelt mittels lokaler Therapie. 1996 wurde der damals 20-jährige Patient erstmals vorstellig in der Pneumologischen Klinik unseres Hauses aufgrund einer Trachealstenose infolge der trachealen Papillomatose. Durch die Insertion eines Trachealstents konnte die Trachealstenose überbrückt werden.

\section{Verlauf}

Seit dieser Zeit musste der Patient $1 / 4$-jährlich zur stationären Behandlung aufgenommen werden, da immer wieder rezidivierende symptomatische Trachealstenosen entweder proximal oder distal des Stents auftraten. Es erfolgte die Abtragung der Papillome lasergestützt und zum Teil wurden Trachealstents in andere Lagen positioniert bzw. in einer anderen Länge. Im Verlauf wurde es dennoch möglich, den Trachealstent zu entfernen und ausschließlich eine Laserbehandlung durchzuführen bzw. die Läsionen mechanisch endobronchial abzutragen. Therapieversuche in Form einer lokalen Applikation von 30 Mio. IE Roferon oder eine lokale Instillation von Cidofovir waren nicht erfolgreich. Eine maligne Transformation der Papillomatose konnte histologisch ausgeschlossen werden. Rezidive waren jeweils nach 8 Wochen erneut behandlungspflichtig ( $\bullet$ Abb. 1).

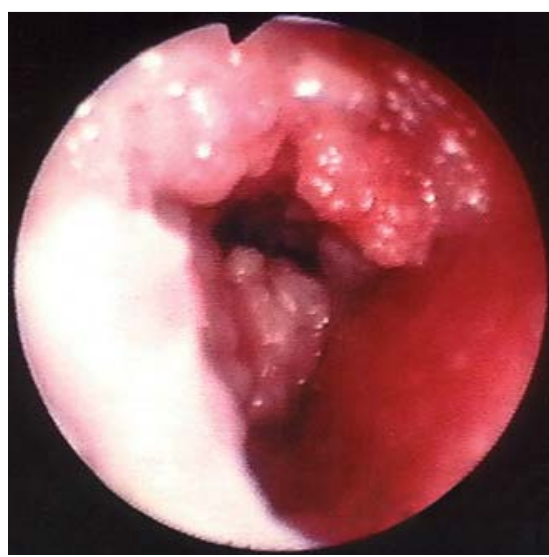

Abb. 1 Bronchoskopiebefund: tracheale Papillome, 2007.

\section{Therapieentscheidung}

Im Dezember 2007 erfolgte ein Therapieversuch mit dem Angiogenesehemmer Bevacizumab (10 mg/kg KG alle 3 Wochen). Die Therapie wurde gut toleriert. Nebenwirkungen, wie z.B. Blutung, Proteinurie oder Hypertonus, die in der Literatur beschrieben sind, traten nicht auf. Nach Applikation von 6 Zyklen Bevacizu- 


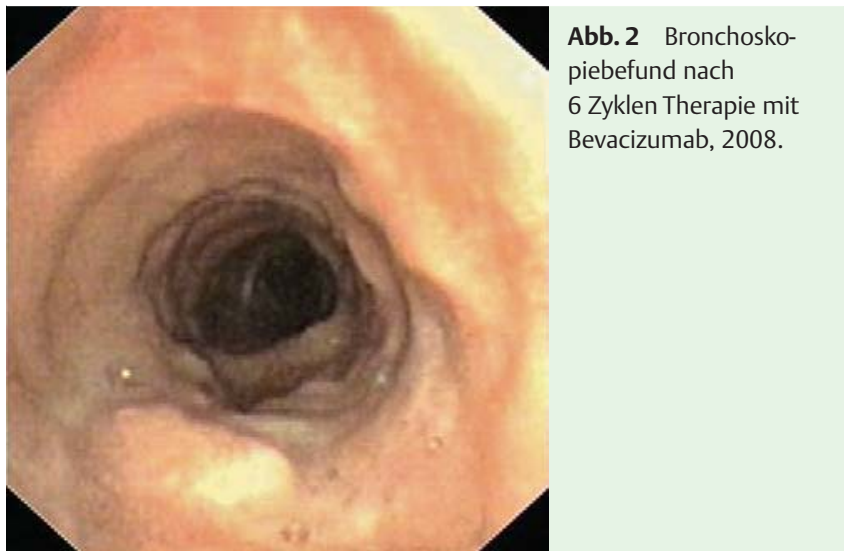

mab war endoskopisch eine eindrucksvolle Rezidivprophylaxe festzustellen ( $\bullet$ Abb. 2). Es fand sich in diesem Zeitraum kein erneutes Papillomwachstum, während in dem vortherapeutischen Zeitraum alle 8 Wochen die Laserbehandlung wegen schwerster Atemnot durch stenosierendes Papillomwachstum notwendig war. Damit ging auch eine deutliche Verbesserung seiner Lebensqualität einher. Bis jetzt erhielt der Patient 8 Zyklen dieser Therapie bis zum Sommer 2008. Seit dieser Behandlung ist der Patient in einer anhaltenden Remission und es kam zu keiner erneuten endobronchialen Papillomabtragung.

\section{Diskussion}

In dieser Kasuistik konnte gezeigt werden, dass durch die Anwendung des VEGF-Inhibitors Bevacizumab eine stabile Regredienz der respiratorischen Papillomatose erreicht werden konnte. Als entscheidender Wirkmechanismus dürfte die Hemmung der Angiogenese angenommen werden. Wie in der Publikation von Rahbar et al. beschrieben wurde, war bei Patienten mit RRP eine vermehrte Expression von vascular endothelial growth factor festzustellen [17]. Das erscheint auch pathophysiologisch nachvollziehbar, da schnell wachsende neue Papillome, wie sie bei dem vorliegendem Patienten über viele Jahre beobachtet werden konnten, auch eine erneute Blutzufuhr brauchen. Deswegen schien die Hemmung der Neoangiogenese für diesen Patientin vielversprechend zu sein. Das ausgesprochen positive Ergebnis mit der langdauernden Remission der RRP, ohne dass weitere Lasertherapien durchgeführt werden mussten, gibt diesem Ansatz recht. Damit steht für die Behandlung der RRP eine vielversprechende neue Therapieform zur Verfügung.

\section{Literatur}

1 Derkay CS. Task force on recurrent respiratory papillomas. A preliminary report. Arch Otolaryngol Head Neck Surg 1999; 121: 1386-1391

2 Dickens P, Srivastava G, Loke SL et al. Human papillomavirus 6, 11, and 16 in laryngeal papillomas. J Pathol 1991; 165: 243-246

3 Armstrong LR, Derkay CS, Reeves WC. Initial results from the national registry for juvenile-onset recurrent respiratory papillomatosis. RRP Task Force. Arch Otolaryngol Head Neck Surg 1999; 125: 743 - 748

4 Wiatrak BJ, Wiatrak DW, Broker TR et al. Recurrent respiratory papillomatosis: a longitudinal study comparing severity associated with human papilloma viral types 6 and 11 and other risk factors in a large pediatric population. Laryngoscope 2004; 114: 1 -23

5 HPA. Trends in anogenital warts and anogenital herpes simplex virus infection in the United Kingdom: 1996 to 2005. CDR Wkly 2006; 16: $1-4$

6 Silverberg MJ, Thorsen P, Lindeberg H, Grant LA, Shah KV. Condyloma in pregnancy is strongly predictive of juvenile-onset recurrent respiratory papillomatosis. Obstet Gynecol 2003; 101: 645-652

7 Kashima HK, Shah F, Lyles A et al. A comparison of risk factors in juvenile-onset and adult-onset recurrent respiratory papillomatosis. Laryngoscope 1992; 102: 9-13

8 McKaig RG, Baric RS, Olshan AF. Human papillomavirus and head and neck cancer: epidemiology and molecular biology. Head Neck 1998; 20: $250-265$

9 Kashima H, Mounts P, Leventhal B et al. Sites of predilection in recurrent respiratory papillomatosis. Ann Otol Rhinol Laryngol 1993; 102: 580-583

10 Evander M, Frazer IH, Payne E et al. Identification of the alpha6 integrin as a candidate receptor for papillomaviruses. J Virol 1997; 71: 24492456

11 Joyce JG, Tung JS, Przysiecki CT et al. The L1 major capsid protein of human papillomavirus type 11 recombinant virus-like particles interacts with heparin and cell-surface glycosaminoglycans on human keratinocytes. J Biol Chem 1999; 274: 5810-5822

12 Bedell MA, Hudson JB, Golub TR et al. Amplification of human papillomavirus genomes in vitro is dependent on epithelial differentiation. J Virol 1991; 65: 2254-2260

13 Zeitels SM, Akst LM, Burns JA et al. Office-based 532-nm pulsed KTP laser treatment of glottal papillomatosis and dysplasia. Ann Otol Rhinol Laryngol 2006; 115: 679-685

14 Stamataki S, Nikolopoulos TP, Korres S et al. Juvenile recurrent respiratory papillomatosis: still a mystery disease with difficult management. Head Neck 2007; 29: 155-162

15 Spiegel JH, Andrus JG, Stefanato CM et al. Histopathologic effects of cidofovir on cartilage. Otolaryngol Head Neck Surg 2005; 133: 666-671

16 Reck $M$. Antiangiogenese beim fortgeschrittenen, inoperablen nichtkleinzelligen Lungenkarzinom (free). Thieme Refresher Onkologie 2008; 5: R25-R44

17 Rahbar R, Vargas SO, Folkman $J$ et al. Role of vascular endothelial growth factor-A in recurrent respiratory papillomatosis. Ann Otol Rhinol Laryngol 2005; 114: 289-295

\section{Interessenkonflikte}

$\nabla$

Keine angegeben 